\title{
A Novel Stateless Energy-Efficient Routing Algorithm for Large-Scale Wireless Sensor Networks with Multiple Sinks
}

\author{
Mujdat Soyturk, Turgay Altilar \\ Istanbul Technical University, Department of Computer Engineeing \\ Istanbul, Turkey \\ msoyturk@dho.edu.tr, altilar@itu.edu.tr
}

\begin{abstract}
As the energy becomes the most valuable resource in the wireless sensor networks, to prolong the lifetime of the network, energy-aware protocols are proposed which use the energy minimization techniques and multi-hop paths. However, multi-hop paths and complex calculations at nodes introduce delay and processing overhead. Providing energy-efficiency becomes a scalability problem. Scalability of a routing protocol for wireless sensor networks is mainly affected by the topological changes and by the number of sensor nodes in the network. In large scale networks with a large number of sensor nodes, multiple-sinks (gateways) should be used to provide scalability. In this paper, a novel stateless energy-efficient routing technique, Stateless Weight Routing with Multiple Sinks (MS-SWR), is introduced for wireless sensor networks. MS-SWR requires no local/global topological information. Property of being stateless avoids the communication overhead and reduces the calculations to minimum. In MS-SWR, reliability is provided by using multiple paths which also reduces the delay to minimum for the time-critical real-time traffic. The performance evaluation of the proposed technique shows that MS-SWR is scalable for large scale wireless sensor networks with multiple sinks.
\end{abstract}

\section{INTRODUCTION}

In wireless sensor networks, energy becomes one of the most valuable resources, as the network size increases. There are energy aware protocols in the literature, generally using multi-hop paths to use the energy more efficiently. However, increase in the hop number between the source and the destination nodes bears some issues that must be considered [1], [2]. First, nodes close to the sink deplete their energies quickly; leaving the sink unreachable and the system into off-state [3]. Secondly, increase in the hop-number cause more nodes to buffer the packet on-the-route, causing a processing overhead and delay at nodes. Processing overhead and buffer fill-up may cause the packets to be dropped. On the other hand, delay at nodes challenges with the real-time requirements of the system [1].

As the network size grows, the length of the constructed paths will increase, causing the problem described above more challenging. On the other hand, the energy consumption will not be efficient anymore. The delay will increase, and the packets will be dropped. Packet drops will cause retransmissions, which increase the delay excessively.

Multiple sinks (multi-sink) usage appears as a solution for large scale networks [1], [3]. However, deploying more sink nodes does not solve the problem directly and evenly. Energy-efficient protocols should be adapted for the multi-sink networks. However, the protocol in use may not be energy-efficient anymore in large scale networks due to increase in the number of nodes. Tablebased protocols fall into this category. Due to topological changes, keeping up-to-date local/global routing tables at nodes makes them inefficient in routing process. Tablefree protocols should be used to provide energy-efficiency and scalability. Routing without tables can be achieved by using geographic routing protocols.

Geographic routing protocols use greedy scheme or beaconless scheme for routing. In greedy schemes [4]-[7], nodes select the best next node on the route by using the local topology information. Collecting local topology information in greedy schemes consumes more energy than beaconless schemes due to reduced transmissions in the latter one. On the other hand, beaconless routing protocols in the literature propose solutions to be implemented at the MAC layer [8]-[12]. In those solutions, RTS and CTS packets are also used for implementing routing protocol that increases the complexity of the MAC layer. Besides that, those solutions become dependent to the MAC layer they use.

Related studies in multi-sink sensor networks [1]-[3], [13]-[17] do not propose a novel routing algorithm. In this paper, we propose a novel stateless routing algorithm for large scale wireless sensor networks, which also works 
with multiple sinks. The proposed algorithm is based on the data flow approach proposed in [18]. The proposed stateless routing algorithm is completely MAC-layer independent, being also the first one in the literature. Nodes do not have to be aware local or global topology information. Routing is achieved without keeping tables. Nodes' geographical positions are sufficient for routing process.

The proposed algorithm, MS-SWR, has the following properties, some of which are properties of SWR;

- MS-SWR provides scalability by not using routing tables, and by not beaconing.

- MS-SWR simplifies routing process by using a weight metric, and designing an appropriate algorithm for routing.

- MS-SWR decreases calculations, delay, and resource requirements (such as processor and memory) at nodes by using weight metric.

- MS-SWR decrease energy consumption by not beaconing, by using position-based routing based on threshold and considering the energy levels of the nodes.

- MS-SWR provides reliability by using multiple paths.

- MS-SWR executes routing process completely in network layer, independent from the MAC layer used below.

- MS-SWR can be used with multiple sinks without any modification in the protocol.

- MS-SWR is scalable in large-scale sensor networks.

The remainder of the paper is organized as follows. In the next section, we review the related works and existing forwarding schemes for geographical routing. In section 3 , we give the approaches and algorithms for the routing algorithm. Performance evaluations are given in Section 4. In the last section we conclude the paper.

\section{RELATED WORK}

There is a little work done on the multiple-sink wireless sensor networks. In [14], multiple sink location problems to manage the energy efficiently and solutions to these problems are presented. In [15], repositioning of the sink node to enhance the performance metrics is investigated. In [16], the formulation to find optimal locations of multiple sinks is proposed. Reference [3] proposes a solution for correlated data gathering to minimize the system-wide energy consumption. In [1], the worst case analysis of sensor networks with multiple sinks, namely, network calculus is presented. Reference
[13] presents a methodology for optimally designing the topology to optimize the communication cost for wireless sensor networks with multiple sinks. Reference [2] proposes a model to adopt existing single-sink algorithms to multi-sink networks. Reference [17] proposes a twotier data dissemination approach for large-scale sensor networks, which is completely proactive and energyinefficient.

\section{STATELESS WEIGHT ROUTING FOR LARGE-SCALE MULTI-SINK SENSOR NETWORKS: MS-SWR}

We propose a new novel stateless energy-efficient routing algorithm for large scale wireless sensor networks. As far as we now, it is the first stateless protocol in the literature that works with multiple sinks in large-scale sensor networks. MS-SWR is a completely distributed stateless algorithm that does not require priori topology information. No routing table and beacon messaging is used. The protocol works with any number of sink nodes, as increase in the number of sink nodes enhance the performance results. The protocol is based on the data flow approach and the routing algorithm (SWR) proposed in [18] which is completely executed in the network layer and is independent from the MAC layer, what ever used. Besides that, MS-SWR constructs multiple braided paths for robustness with a minimum delay and provides a basis for real-time support for timecritical data.

Routing could have been completely achieved by using geographical positions. However, instead of geographical positions, we use another value, namely weight value. Each node derives its weight value dynamically from its current position. There are two main reasons for using weight values: first, it aids to routing process and makes it simple to implement, and secondly, it minimizes delay, energy consumption, and processing requirements at nodes in routing decision phase.

The weight function takes the location information (e.g. geographical position, or relative position such as (x, y)) as input and produces the weight value. The weight function can be optimized to optimize the network metrics and the network parameters such as network-lifetime, node lifetime, emergency conditions, silence, etc. Therefore, while providing the routing, some other parameters can be optimized. A simple weight function can be as simple as the following one:

$$
f(x, y)=x^{2}+y^{2}
$$

When a node has data to transmit, inserts its and the destination's weight values into the packet, and broadcasts the packet. When a node receives a packet, it 
compares its weight value with the weight values in the packet. If its weight value is between the transmitting node's weight value and the destination's weight value, it rebroadcasts the packet, or drops the packet otherwise. If the nodes in the operation area are uniformly distributed, less than half of the nodes in the range of the transmitting node rebroadcast the packet. To reduce the number of rebroadcasting nodes, a threshold value is used and inserted into the packet. Only the nodes those have weight difference greater than the threshold value can rebroadcast the packet. By this way, nodes closer to transmitting node are avoided to rebroadcast. Rebroadcasting nodes are those that make more advances toward the destination..

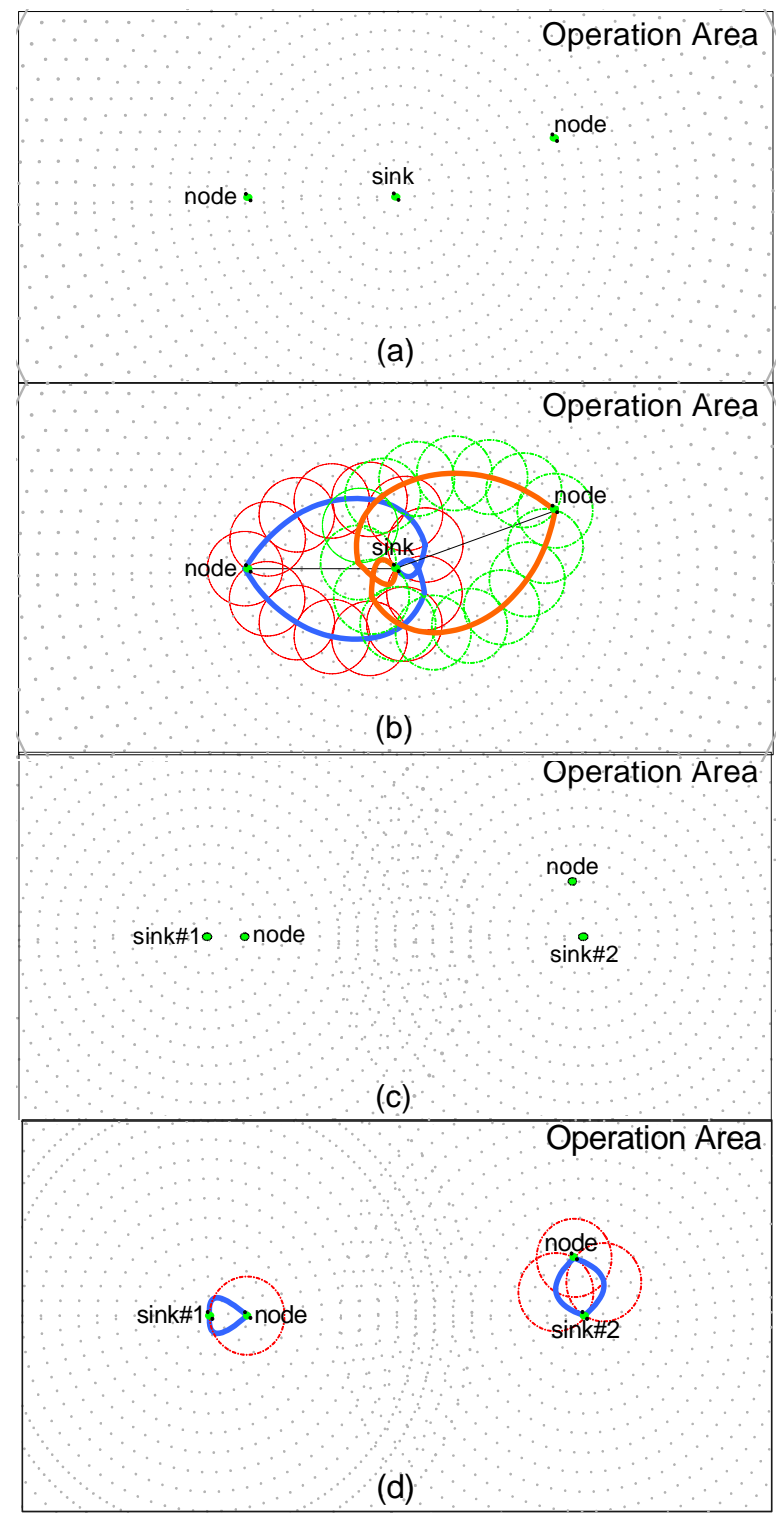

Fig. 1 Multi-sink usage reduces the path lengths with respect to the single sink usage.

\subsection{Multiple Sink Deployment}

MS-SWR protocol can be applied in a network with any number of sinks. Number of the sinks does not affect the protocol. No modification is required to the protocol. However, increasing the number of sink nodes causes the network to perform better results. Sinks can be positioned anywhere in the network. We assume that each sink informs the neighborhood nodes about its position. In a static network, this information can be diffused only once, e.g. on the node deployment phase.

MS-SWR can also be applied in mobile sensor networks. In mobile networks, a sink must inform the sensor nodes in the neighborhood about its current position, as the position of the sink changes. Furthermore, sinks may move to new locations to optimize the coverage area and the performance metrics. Sensor nodes set the closest sink's position as the origin $(0,0)$, and evaluate their own positions relative to the sink's position. This is achieved only once at the node deployment phase.

In Fig. 1(a), a single sink is positioned in the center of the operation area. Two nodes have data to send to the sink. The possible relaying nodes are those that are located in the two symmetric logarithmic spiral curve shaped area (Fig. 1(b)). More than one path will be constructed toward the sink from each node. Distance of the nodes to the sink affects the size of the symmetric logarithmic spiral curve shaped area, which therefore affects the number of transmissions. Two sinks are positioned optimally to cover the operation area in Fig.1(c). As the distances to sink get closer, number of transmissions decreases. We emphasize that MS-SWR is a stateless protocol and energy is consumed only on events. However, table-based protocols consume a great amount of energy, even if there is not any data to send.

\section{SIMULATIONS}

\subsection{Simulation Parameters}

In this section we present our simulation results. There is no packet loss due to transmission collisions in or simulation environment. We use the parameters given in [19] to make the results comparable with the proposed evaluations. To provide the double range property, nodes have a sensing range $\left(R_{s}\right) 50 \mathrm{~m}$ and a transmission range $\left(R_{c}\right) 100 \mathrm{~m} \quad\left(R_{C} / R_{s}=2\right)$. Fifty nodes are uniformly distributed in a well-defined topology [20] over an area $300 \mathrm{~m} \times 500 \mathrm{~m}$. Network is designed with the methodology defined in [19] and nodes randomly generate packets with a probability of $0.05 \mathrm{pkt} / \mathrm{min}$. Destination (gateway or sink) nodes are positioned uniformly in the operation are. We compare the proposed approach with the flooding and "GPSR without perimeter" algorithm. Parameters for GPSR are obtained from the results of [19]. Default 
threshold value is set to Rc/2 for MS-SWR protocol. The proposed results are the averages of 10 runs of 900 seconds simulation periods. Energy consumption ratio for idle/receiving/transmitting states is 1/1.05/1.4. 1000 joule is given to each node at the startup of the simulation.

We mainly focus on the energy consumption. Detailed results are obtained as the energy consumption in transmission and receive processes for the routing, measurement of the network lifetime, comparison of the remaining energies of the nodes and the system.

\subsection{Remaining Energy}

Table 1 Comparison of the protocols.

\begin{tabular}{|c|c|c|c|}
\hline & Flooding & GPSR & $M S-S W R$ \\
\hline $\begin{array}{l}\text { Average System } \\
\text { Lifetime }\end{array}$ & $141 \mathrm{sec}$ & $\begin{array}{l}139 \\
\text { sec }\end{array}$ & $>900 \mathrm{sec}$ \\
\hline $\begin{array}{l}\text { Time of the First } \\
\text { Node Termination }\end{array}$ & $136 \mathrm{sec}$ & $\begin{array}{l}132 \\
\text { sec }\end{array}$ & $\begin{array}{l}\text { NONE } \\
\text { in 900sec }\end{array}$ \\
\hline $\begin{array}{l}\text { Average Number of } \\
\text { Terminated Nodes } \\
\text { on Destination } \\
\text { Unreachable }\end{array}$ & 65 & 9 & $\begin{array}{l}\text { NONE } \\
\text { in 900sec }\end{array}$ \\
\hline
\end{tabular}

We deployed different number of sink nodes in the first scenario to observe how the routing protocols are affected from multiple-sink deployment. We observed both the remaining energy level of the system and the remaining energy levels of the nodes. Fig. 2 shows the remaining energy levels of the system with a single sink in simulation 1. GPSR and the flooding protocol deplete the system energy very quickly. In GPSR, the simulation ends after 139 seconds failing to find routes. The overall system energy of the GPSR protocol is a little better than flooding, causing the system to live longer than flooding. GPSR depletes most of its energy at the beaconing, while the flooding depletes its energy on routing process. The observed system energy in GPSR protocol is according to the beaconing period with 1 sec. The system will live longer in GPSR protocol when the beaconing interval is extended. MS-SWR protocol continues to live when the simulation ends after 900 sec. The remaining system energy in MS-SWR is higher than GPSR and flooding for each second. In MS-SWR, the energy is consumed only in routing processes. The energy consumption decreases linearly in MS-SWR. In MS-SWR, none of the nodes terminates at the end of the simulation (Table 1).

We observed that increasing the number of the sinks does not affect the performance of the flooding protocol and the GPSR protocol. Adding more sinks does not avoid the transmissions in flooding. On the other hand, in GPSR, increase in the number of the sinks only affects the transmissions in data packets. The energy is mainly consumed in beaconing. Decreasing the shortest path in
GPSR only avoids a few transmissions. However, in MSSWR, shortening the distance between the source node and the sink node, decreases the area. Therefore, using multiple sinks decrease the energy consumption in MSSWR (Fig. 3). Similar results are observed in the Scenario 2. Flooding and GPSR protocols are depleted their energies very quickly and adding more sinks did not effect the energy consumption in these protocols. However, the flooding protocol consumed all the system energy in a few seconds. As seen in Fig. 4 that adding more sink nodes in MS-SWR increases the system energy. Remaining energy levels of the nodes is given in Fig. 5. Nodes preserve their energy levels. Almost all of the 1600 nodes have remaining energy levels higher than $80 \%$, when the simulation ends at 900 sec. However, GPSR protocol fails to live at 139 sec.

\section{CONCLUSIONS}

In this paper, we propose a novel stateless energyefficient routing algorithm (MS-SWR) for wireless sensor networks with multiple sinks. MS-SWR is based on the SWR protocol. We introduce the weight metric to enable the table-free routing. Using the weight metric also reduces the total number of calculations at nodes drastically, and also minimizes delay, energy consumption, and resource requirements such as processor and memory at nodes. Routing is achieved completely in network layer according to the ISO OSI Reference model. Being independent of the MAC-layer makes MS-SWR applicable with any MAC-layer below it. This feature makes it unique while the other stateless routing protocols in the literature propose MAC-layer involved solutions. MS-SWR works with any number of sink nodes without any modification in the protocol. We demonstrated that without any topology information, MSSWR forwards the packet to the destination over multiplepaths to provide reliability. Performance results show that the MS-SWR prolongs the network lifetime longer than flooding and GPSR, and has lower energy consumption. On the other hand, MS-SWR provides a basis for realtime traffic by means of delay and reliability. Usage of the weight metric and the stateless property enable the MS-SWR to be applicable in mobile networks. Future research includes the implementation of mobility patterns to the sinks and the sensor nodes.

\section{REFERENCES}

[1] J.B. Schmitt, F.A. Zdarsky, U.Roedig. "Sensor Network Calculus with Multiple Sinks", In Proceedings of the 11th IFIP International Conference on Personal Wireless Communications (PWC'06), Albacete, Spain. Springer LNCS, September 2006.

[2] A. Das, D. Dutta. "Data Acquisition in Multiple-sink Sensor Networks", Mobile Computing and Communications Review 9(3): 82-85 (2005) 
[3] K. Yuen, B. Liang, B. Li. “A Distributed Framework for Correlated Data Gathering in Sensor Networks”, IFIP 2006

[4] Karp, H.T. Kung, "GPSR: Greedy Perimeter Stateless Routing for Wireless Networks”. Mobicom 2000, (2000)

[5] S. Giordano, I. Stojmenovic, L. Blazevic. "Position Based Routing Algorithms For Ad Hoc Networks: A Taxonomy”, in: 'Ad Hoc Wireless Networking', X. Cheng, X. Huang and D.Z. Du (eds.), Kluwer, 2004, 103-136

[6] M. Mauve, J. Widmer, H. Hartenstein. "A Survey on Position-Based Routing in Mobile Ad-Hoc Networks", IEEE Network Magazine, 15(6):30--39, November 2001

[7] F. Araujo, L. Rodrigues. "Survey on Position-Based Routing”, TR-01, University of Lisbon, (2006)

[8] H. Fuessler, J. Widmer, M. Kasemann, M. Mauve. "Beaconless Position-Based Routing for Mobile Ad-Hoc Networks", TR-03-001, Dept. of Comp. Science, University of Mannheim, February 2003

[9] Blum, T. He, S. Son, J. Stankovic. "IGF: A State-Free Robust Communication Protocol for Wireless Sensor Networks”, Technical Report CS-2003-11, University of Virginia, Charlottesville, VA. 2003.

[10] M. Heissenbuttel, T. Braun, "A Novel Position-based and Beacon-less Routing Algorithm for Mobile Ad Hoc Networks", ASWN'03, Bern (2003), 197-210

[11] M. Chawla, N. Goel, K. Kalaichelvan, A. Nayak, I. Stojmenovic, "Beaconless Position Based Routing with Guaranteed Delivery for Wireless Ad-Hoc and Sensor Networks”, IFIP 1st Int.Conf.On Ad-Hoc Networking at 19th IFIP World Computer Congress, Santiago, Chile, August, (2006) 20-25

[12] M. Heissenbuttel, T. Braun, M.Walchli, T.Bernoulli. "Optimized Stateless Broadcasting in Wireless Multi-hop Networks”, IEEE Infocom 2006, Barcelona, 23-29 Apr 06.

[13] M. Kalantari, M. Shayman. "Design Optimization of MultiSink Sensor Networks by Analogy to Electrostatic Theory", WCNC 2006.

[14] E.I. Oyman, C. Ersoy. "Overhead Energy Considerations for Efficient Routing in Wireless Sensor Networks", Computer Network, Vol.46, pp. 465-478, 2004

[15] Akkaya, M. Younis, M. Bangad, "Sink Repositioning for Enhanced Performance in Wireless Sensor Networks". Elsevier Computer Networks Journal Vol.49/4 (2005) 512-534

[16] H. Kim, Y. Seok, N. Choi, Y. Choi, T. Kwon: "Optimal Multi-sink Positioning and Energy-Efficient Routing in Wireless Sensor Networks.” ICOIN 2005: 264-274

[17] H. Luo, F. Fe, J. Cheng, S. Lu, L. Zhang. “ TTDD: A TwoTier Data Dissemination Model for Large-scale Wireless Sensor Networks”, ACM/Kluwer Mobile Networks and Applications (MONET), Special Issue on ACM MOBICOM

[18] M. Soyturk, T.Altilar. "Source-Initiated Geographical Data Flow for Wireless Ad Hoc and Sensor Networks", IEEE WAMICON 2006.

[19] D. Son, A. Helmy, B. Krishnamachari. "The Effect of Mobility-Induced Location Errors on Geo.Routing in Mobile Ad Hoc and Sensor Networks: Analysis and Improvement Using Mobility Prediction”, IEEE Trans. Mobile Comput.Vol.3, No.3, Jul-Sep, 233-245 (2004)

[20] C. Avin. "Fast and Efficient Restricted Delaunay Triangulation in Random Geometric Graphs", Workshop on Combinatorial and Alg. Aspects of Networking, 2005

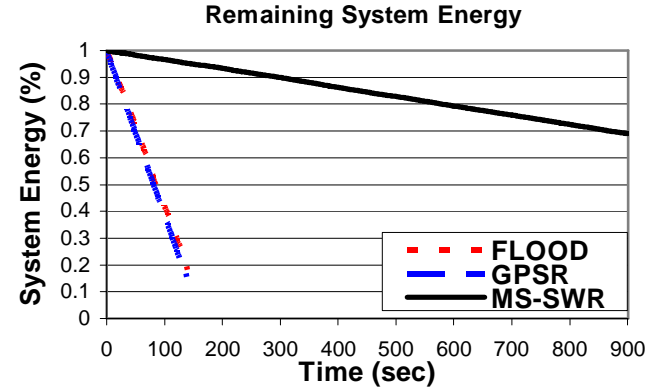

Fig. 2 Remaining energy levels of the protocols in Scenario 1 with a single sink.

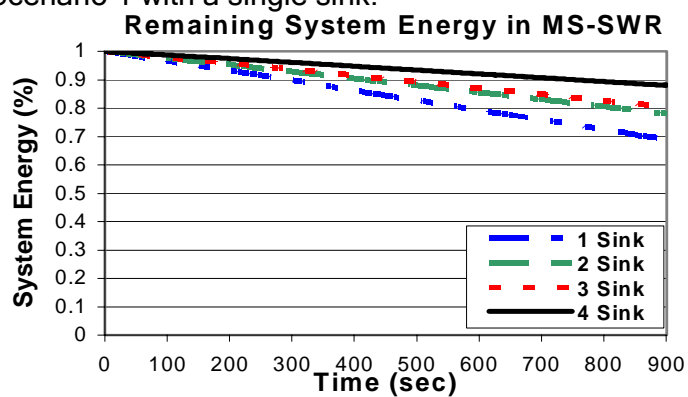

Fig. 3 Remaining system energies of the network when the MS-SWR protocol is used with multiple sinks in Scenario 1.

Remaining System Energy in MS-SWR

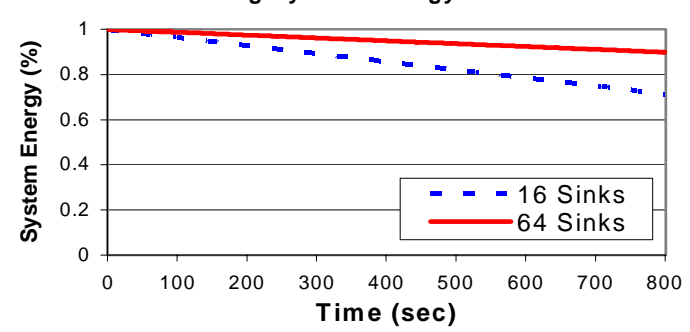

Fig. 4 Remaining system energies of the network when the MS-SWR protocol is used with multiple sinks in Scenario 2 .

Remaing Energy Levels Of Nodes in MS-SWR

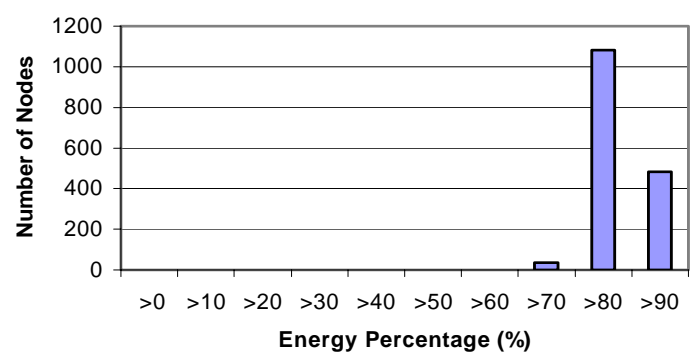

Fig. 5 Remaining Energy levels of the nodes in Scenario 2 , when the MS-SWR protocol is used. Nodes preserve their energies in a large-scale network. Almost all of the nodes have energy levels higher than $80 \%$, when the simulation ends at $900 \mathrm{sec}$. 